\title{
Open Educational Resources: The Role of OCW, Blogs, and Videos in Computer Networks Classroom
}

http://dx.doi.org/10.3991/ijet.v7i3.2116

P. Gil, F.A. Candelas, G.J. García, C.A. Jara

University of Alicante, Alicante, Spain

\begin{abstract}
This paper analyzes the learning experiences and opinions obtained from a group of undergraduate students in their interaction with several on-line multimedia resources included in a free on-line course about Computer Networks. These new educational resources employed are based on the Web 2.0 approach such as blogs, videos and virtual labs which have been added in a web-site for distance self-learning.
\end{abstract}

Index Terms - Educational Resources; OpenCourseWare; Blogs, Videos and Simulations.

\section{INTRODUCTION}

The work presented in this paper describes the implementation and results in learning of a set of educational resources for an open and free education. This type of resources is designed for an independent learner and/or a distance self-learning. The process of convergence to ESHE (European Space of Higher Education) [1] determines that traditional education must be combined with distance education because a good distance teaching education provides flexibility to students. This way, the students can configure their own free schedule according to their necessity and level of knowledge. The distance education requires of academic rigor and it can be achieved with monitoring and supervision by the teacher, a good organization where lessons are correctly focused to the course objectives and where the students have original and suitable educational resources to learn. The Open Educational Resources (OERs) [2] play an important role in this new educational context. In previous works, LCMS (Learning Content Management System) like Moodle and virtual laboratories based on KivaNS+EJS have been designed and implemented $[3,4]$ to improve the learning by means free and open software. Nowadays, two new resources have been used to the educational process: OpenCourseWare and blog sites. Their description and their influence are studied in this paper.

The MIT OpenCourseWare (OCW) project [5] has significantly improved the distance/on-line learning and teaching [6]. The OCW is a free open digital publication of educational materials, organized as courses. In May 2011, the University of Alicante (UA) won the award of OCW-Consortium 'Landmark Site' for excellence. In this paper, the Open Course of the subject "Computer Networks" [7] which was launched in 2010 as part of OCW-UA, is described. Thus, this paper describes what
OERs have been included in the Open Course of Computer Networks, such as textbooks, video logs, tests, software, links, and other tools or techniques used to support access to knowledge about Computer Networks. In addition, some studies have been done to collect the opinion of students and new or potentials teachers (lectures) respect to Open Course of Computer Networks. Other instruments, as monitoring access, have also been used to characterize the visits and the visitors. However, the OCW is not the only way to distribute educational resources. Other booming tools are Blogs $[8,9]$ and Wikis [10].

Furthermore, in this paper we will present a discussion about the use and usability of the blog of the subject "Computer Networks" [11] and its contents. The students have been asked if the blog satisfies their expectations. In this work, it is also described the student experiences when they require search information about the subject such as tutoring schedules, recommended books, free software to download, new online courses as OCW belonging to other universities and other educational resources. Thus, the content of the site and its structure and organization will be discussed.

The perception about the educational resources is a very relevant issue. New educational resources such as multimedia, video, web site, OCW, etc. must be attractive. In the frame of the work presented here, the students were asked about it in order to develop new improvements. Together with OCW for Computer Networks subject, a blog for the subject has been created. This blog has been voted winner as the best 2011-blog of University of Alicante. It has been awarded due to its quality of learning resources, structure, organization and contents.

The paper is organized as follows: Section II describes the blog and the OCW implemented for the Computer Networks subject and discusses why using a blog as an educational tool. Also, it explains how the OCW influences in the teaching and learning methodology. Furthermore, the opinion of students about the blogs in the learning process is shown in this section. Afterwards, Section III comments other digital resources used to improve learning such as streaming video. Finally, some important conclusions are reported in Section IV. 


\section{THE BLOG AND OCW}

\section{A. What is the blog and/or OCW for?}

In April 2001, MIT announced the OpenCourseWare (OCW) project [5]. MIT defined an OCW as course materials that are used in the teaching of almost all undergraduate and graduate subjects available on the web, free of charge, to any user anywhere in the world. It is important to remark that the educational material of an OCW must get from existing classroom courses and not for a specially developed e-learning environment.

In our opinion, a courseware should be developed as an integrated part of courses which consist of a multimedia mix of materials (such as textbooks, videos, software, links and interactive simulations) and not only the electronic resources of the theoretical classes with some additional support. Our OCW was created in this way. Students demand the content of an OCW be more interactive, because the slides and files of the subject contents are hard to understand without a context of the course. Fig. 1 depicts an image of the appearance of this OCW's web site [7]. The OERs included in the OCW about the subject of Computer Networks are composed by multimedia videos, electronic documents, interactive simulations and several external links to provide more information to students. In general, the students would prefer short movies or video logs. These make the web site of OCW more active and attractive.

The organization of the OCW has been done following the next structure: Presentation, Aims and Scope, Contents, Educational Methodology, Evaluation, Materials, Bibliography and Internet Resources.

For the Presentation of the subject in the OCW, the teachers developed two educational video logs (for the theoretical and practical part) where the contents, resources and methodology of the subject are explained by means of a presentation embedded in the same video. Aims and Scope, Contents, Educational Methodology and Evaluation are simple and static web pages where the subject organization is explained by the teachers in the real context. In the Materials section, all the textbooks and documents used by the teacher are provided in electronic form and they are linked from the Repository of the University of Alicante [13]. Finally, in the Internet resources section, there are several links to interactive applets developed by the authors [3,4] about computer network simulations.

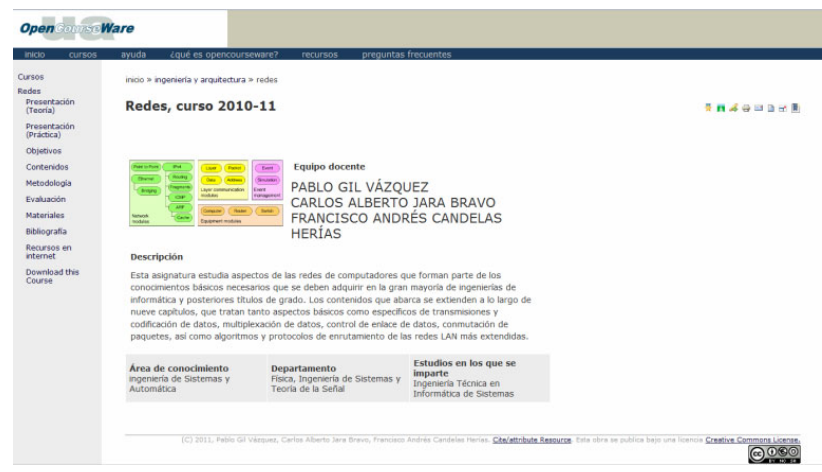

Figure 1. OCW for Computer Networks subject

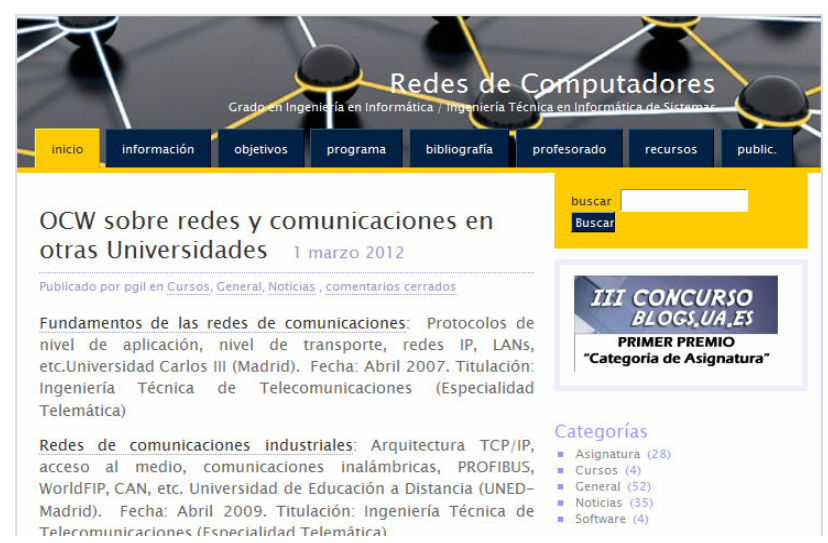

Figure 2. Blog of Computer Networks subject

This OCW of Computer Networks is very useful because it allows students to engage in their self-learning study, providing on-line resources available outside the classroom or laboratory. Additionally, this OCW can be used by other teachers from other universities in order to follow the educational contents and methodology used in the subject, because the course is open.

Regarding the blog, this kind of resource has already been used successfully to enhance the communication between students and teachers in educational contexts [9]. Blogs not only use simple user interfaces but also provide a tool that allows users to easily maintain the content or add new entries. One of the most important features of blogs is that they are not geographically or temporally constrained [14]. In [15], the main activities for learning that can be performed with learning-oriented blogs are summarized in: reading blogs of others, receiving comments and previewing tasks of others and reading feedback received in relation to these. The reasons behind teachers' willingness to adopt teaching blogs have been widely studied in [16]. In this work, Lai and Chen numerate eight significant influences on teaching blog adoption: perceived enjoyment, codification effort, compatibility, perceived ease of use, personal innovativeness, enjoyment in helping others, school support and perceived usefulness, ranked by relative importance.

Blogs allow a different group of people to achieve communication and content production. A blog overcomes the limitations of a LCMS as Moodle, where the platform is closed and it is sometimes used merely as a repository of documents. Blogs provide the ability to broadcast events, news, courses, announcements, jobs, jobs at other universities and institutions, etc.

Our blog provides useful information about Computer Networks subject. There are posts related to the content of the course, reporting the objectives, the program or the bibliography of the subject. Fig. 2 depicts an image of the appearance of the blog developed for Computer Networks subject at University of Alicante [11].

Nevertheless, the blog has not been created to post only basic information related to the subject. Related news, courses or links have been added to attract the student's attention while teachers increase the students learning possibilities. Recently, new posts with embedded short videos, which have been produced by the students themselves, have been added. Each video deals with a particular topic of the practical laboratory work. The posts 
can be commented by other students, generating an interesting feedback to improve their own work. Blogs are effective tools in driving and motivating learning. With them, learning becomes more informal.

The OCW and the blog provide the possibility to locate and supply material resources for learning and help students find relevant content in relation with the theoretical classes. In addition, these e-learning tools can increase the participation and collaborative relationships between the students, since they can share experiences or opinions during the attending classes.

\section{B. Students' Opinion about blog and OCW for 'Computer Network' subject.}

A survey has been arranged to determine the degree of satisfaction of the students in relation to the blogs and OCW as teaching tool for improve the learning process. The survey consisted of 25 questions to evaluate different issues, such as: the level of agree about Blogs and OCW for subjects and videos. Furthermore, in the last academic year, 2011/12, 69 students have coursed this subject (89\% are men and $11 \%$ women). However, only 45 students have voluntary answered the survey about OCW [7] and Blogs [11]. The average age of respondents is 22 years old. These same students have participated in the development of educational videos about simulations.

On the one hand, the students have compared the blog 'Computer Network' (Fig. 2) with other blogs at the University of Alicante (Fig. 3). The comparison has been done from a viewpoint of handling (ease to use), organization (how the contents have been structured and if they are easy to find), update frequency and the relevance of own and alien links and resources. The scale of qualification is from 1 (totally disagree and/or dissatisfied) to 5 (totally agree and/or satisfied).

Fig. 3 shows the results obtained in the survey performed. As it can be seen in this figure, the great majority of students think that the 'Computer Network blog' is better than other blogs known by students. They stand its organization and the relevance of the resources which are linked. The $43.2 \%$ of students voted 4 on a scale between 1 (the lowest) and 5 (the highest) and the $31.8 \%$ of they voted 3 when they were interviewed about the organization. The best satisfaction score is obtained when they give their opinion about the linked educational resources. So, the $22.2 \%$ and $15.9 \%$ of students are completely satisfied with the own and alien resources (they voted 5). Moreover, the $24.4 \%$ and $47.7 \%$ of students think that the score of these resources are 4 ; in addition the $37.8 \%$ and $27.3 \%$ think that this score is 3 . Moreover, Fig. 4 shows an analysis of the distributions shown in Fig. 3. In this case, the graph looks for the value that occurs most frequently in each data set (statistic value of mode). The value mode is always equal or bigger than 3 for all aspects.

On the other hand, the students were asked about if the learning process improves using blogs and OCW and how it improves. According to the opinion about blogs (Fig. 5), the $78.3 \%$ (equal or bigger than a score of 3 ) of students think that the learning is more fun. The score 3 is the value more repeated by students when they give their opinion about the additional information published in the blog, such as news of computer network world, information about courses taught in other universities and institutions, etc. (the $67.3 \%$ of students voted 3 or more).
Moreover, the students consider (they have voted 4 in the great majority and the $78.2 \%$ vote 3 or more) that the digital resources provided (on-line courses, videos, links to simulators and software) and the communications between students and other people interested in the topic of computer networks become more fluid (the $71.7 \%$ of students). They think that the comments about information of blog can greatly enhance and help the communications among students of different universities or the same university.

According to the opinion about OCW (Fig. 6), the results of the research were the followings: The $80.8 \%$ of students think that an OCW could replace the teaching methodology based on masterly lessons (40.4\% have voted 4 and $40.4 \%$ have voted 3).

The great majority of students think that OCW improves the autonomous learning process. Almost $47 \%$ voted 4 , the $20 \%$ have voted 5 and only $19 \%$ think the opposite (have voted 1 or 2 ).

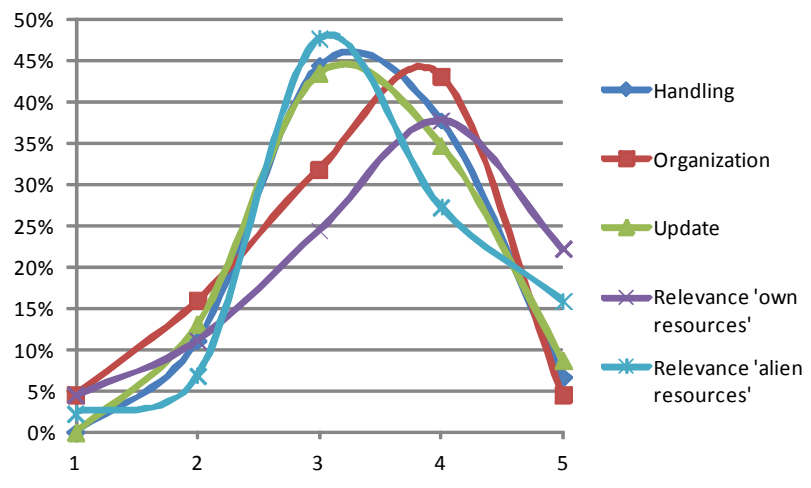

Figure 3. Student's opinion in relation with other blogs of subject

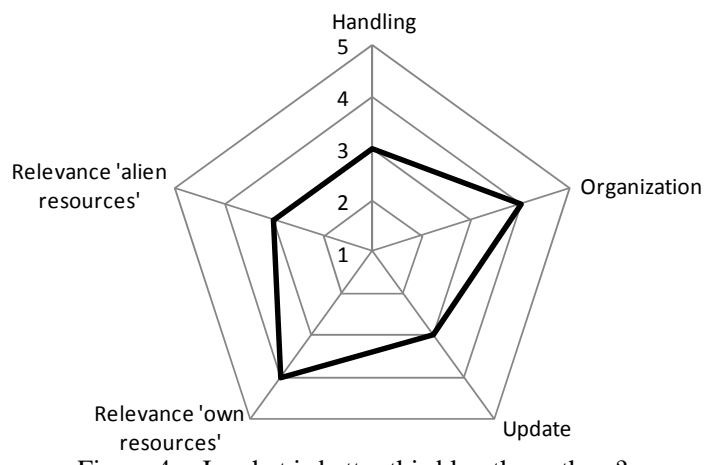

Figure 4. In what is better this blog than others?

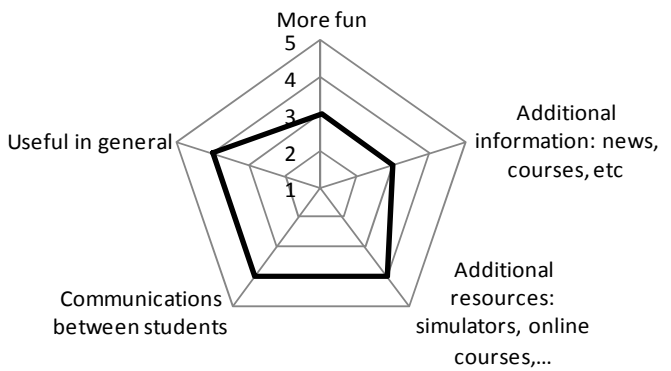

Figure 5. How the learning process improves with Blog 


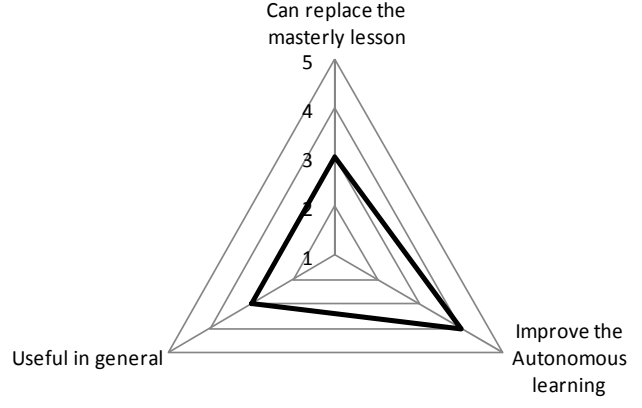

Figure 6. How the process learning improves with OCW

The great majority of students think that OCW improves the autonomous learning process. Almost $47 \%$ voted 4 , the $20 \%$ have voted 5 and only $19 \%$ think the opposite (have voted 1 or 2). In general, the students are now interested in the development of computer-based courseware although the number of these students is lower than the number of students who consider useful the OCW in combination with masterly lesson. In this way, many students think that could be interested only when a distance self-learning methodology is required (53.4\% have voted below 2 and only $13.3 \%$ have voted 4 or more).

The content of the blog is organized in sections. However, these sections are not insulated containers. There are more cross-links that lead to the student from one section to different another. In the blog, the main topics mentioned are organized as general information of subject such as objectives, program, bibliography and teacher information. Furthermore, the different news and courses about computer networks in the world are published and they have been written by other academics. In addition, other kind of educational resources developed by the teachers and by other academics are linked: software, simulators, videos, slides, etc.

The survey had some open questions which the students give their opinion about what sections have been the most and the less visited. The results show that the videos and slides with 22 votes are on the top rate of the resources published from the blog. Other important resources which have been very much accessed are: the notices ( 7 votes) and the detail of the program of practical and theoretical classes (14 votes). The sections with less access were the teacher information, and objectives of subject.

\section{Frequency of student access to Blog and OCW}

When our students are asked about how often they have used the Blog as educational resource, they usually answer 4 times every month. In general, it is one time every week. This access frequency can be observed in Fig. 7. Moreover, in that figure the access comparison with OCW can be observed. Our students usually access once for week (52.3\% and 46.7\%) in both, Blog and OCW. Furthermore, it is remarkable that $33.3 \%$ of students have never accessed to OCW versus the $6.6 \%$ of students who have not accessed to Blog. This fact may be because the blog is a tool more dynamic and flexible in relation to the provided contents and its update frequency. However, the OCW is a static tool and it is more oriented to distance elearning.

The blog, OCW and the links to other websites that these tools contain are not the only educational resources which the student can use throughout the course.
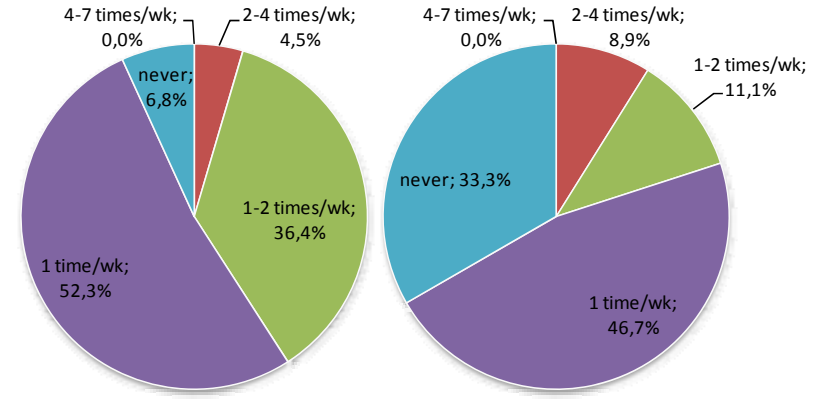

Figure 7. Access frequency of : a) Blog b) OCW

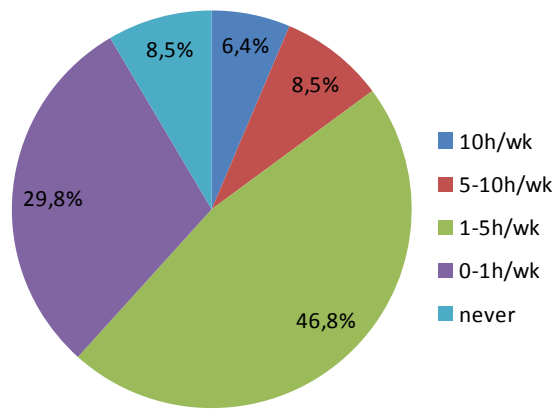

Figure 8. Internet access frequency to consult topics about Computer Networks

For this reason, it is important to emphasize that sometimes the student access to other sites in Internet looking for information in order to clarify doubts, add contents, supplement their learning or simply curiosity.

\section{Frequency of access to Blog and OCW from abroad and other places}

Our OCW materials are accessible since December 2010. Until today, more than 1646 and 4571 users have downloaded materials about theoretical and practical classes, respectively. The OCW visitors come mainly from Spain (47\% and 33.4\%), Latin America (46.1\% and 59.6\%), and North America (3.2\% and 2.7\%), countries where there are an important Spanish speaking community, although, recently, the materials are also being accessed from other geographical areas (1.4\% and 2.6\%), such as Europe, Asia and Africa, with similar percentage. From our University the visits and downloads were $2.3 \%$ and $1.7 \%$. However, the profile of users is unknown. Information about whether they are selflearners, students or educators is not available. Fig. 9 shows the download information classified by type of materials: theoretical lessons and expositions in the classroom and practical lessons and laboratory documents.

On the other hand, our Blog is accessible since June 2010. From February 2011, the monitoring of visits is being done by means of the Google Analytics tool. This tool allows us to know the location and language of visitors from outside of our University. The visitors of the blog are mainly from Spain (35.3\%), Latin America (62\%) and North America (1.2\%), countries where there is an important Spanish speaking community. 
PAPER

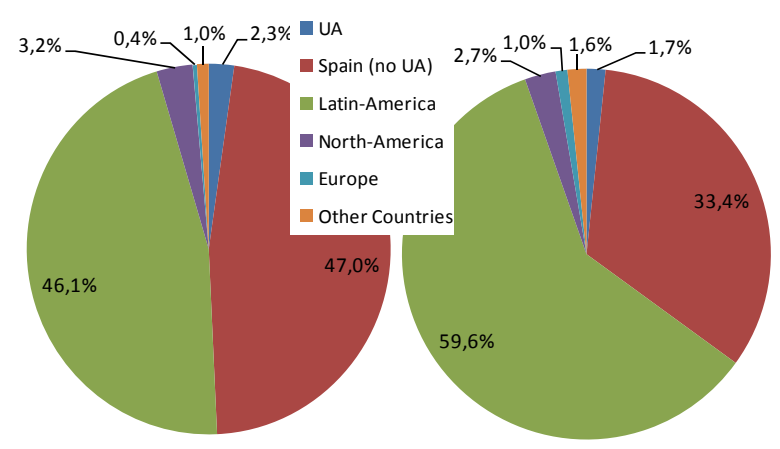

Figure 9. Downloads Distribution of OCW. a) Theoretical lessons. b) Practical lessons

\section{VIDEO LOGS}

The use of videos for documenting laboratory experiences is one of the main aspects on which the proposed methodology is based. This section is centered in describing how this type of resources were created and added in the OCW and blogs, in order to improve the selflearning.

\section{A. What is video logs for?}

Keeping in mind that the direct support of the teacher is not available for the student when he/she studies on their own outside the classroom or laboratory, it was decided to include video logs as new online resources in order to facilitate the understanding about concepts on protocols working. Moreover, this kind of resources can make more productive the self-learning trough the web technologies.

Video logs were proposed as short documentary videos describing the development, step by step, of lab experiences in which the working of a specific protocol is analyzed. In most video logs, the experience is described in the same way that it should be carried out in the laboratory of the faculty, using the same equipment and software tools. In other video logs, concepts are explained by using the network simulator KivaNS [17], which has been developed by the authors' research group.

For example, the Fig. 9 shows two snapshots of a video $\log$ about the operation of ARP and TCP protocols, respectively. In essence, the first video describes the next aspects: the messages which are trigged by the protocol after running the command "ping" in a computer in laboratory, the format of these data messages based on the information captured by a network monitor, and the resulting state in the ARP table of the computer. And the second video describes the segmentation of TCP packets when the MSS value is negotiated between the end nodes in a data transmission process. The route, the size and type of TCP packets and Ethernet frames are described in the process with the support of Wireshark monitor.

Although the first video logs were recorded by the teachers, these soon feel that it would be a good idea that students record their own experiences to share with their peers. Thus, students were encouraged to record video logs to document their laboratory experiments, as an optional activity that scored in the final grade for the course. At the same time, the students who developed videos also reinforced the knowledge that they attempt to explain in videos. The target of OERs, such as video logs, is to enhance the online learning, while, at the same

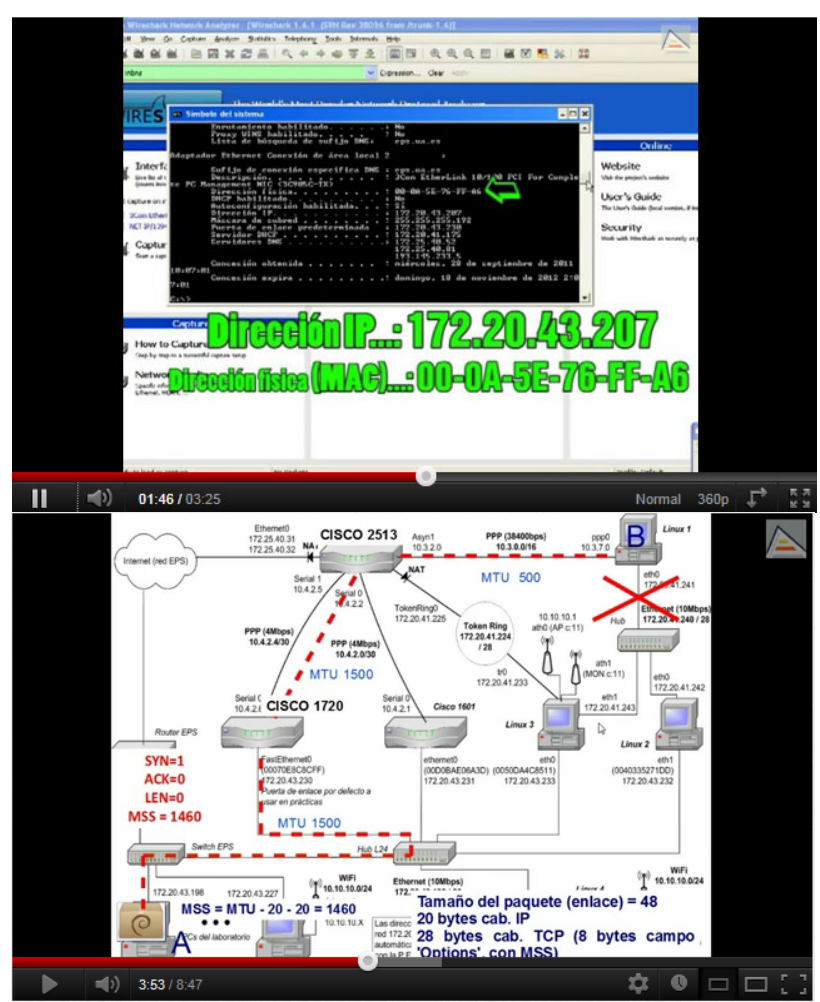

Figure 10. Snapshots of two video logs describing ARP and TCP protocols.

time, the online learner participation is also improved [18].

Teachers have been reviewing the videos produced by students to select those that best describe the different protocols. These selected videos have been published as open online resources on the blog of the subject [11], in addition to the institutional YouTube profile of the University of Alicante, and the popular public Repository of the University of Alicante (RUA) [13].

\section{B. Student's opinion on video logs relevance}

During the last course 2011/12, the Computer Network subject has been taught in two degrees about Computer Sciences. The number of students has been 69 and 196 students, in each career, respectively.

On the one hand, 69 students of a degree were surveyed about OCW and Blogs, and 45 gave their opinions. As commented before, these digital resources were designed not only by the teachers, but also, by the same students, which have participated in the development of other educational resources such as videos logs about simulations and operations of TCP/IP protocols.

On the other hand, in order to obtain an opinion about these new resources, these same videos have been used by other 196 students of another different degree. In this case, 80 students of 196 (93\% are men and only 7\% are women) have opined and evaluated about this new tool as educational videos. It is important that the students who evaluate videos are different to the students who do them. In this case, the average age of respondents is 20 years old. The result of this opinion is shown in the Fig. 10. Five 
PAPER

Open EduCAtional Resources: The Role of OCW, Blogs, AND VideOS In COMPUTER Networks ClassRoOM

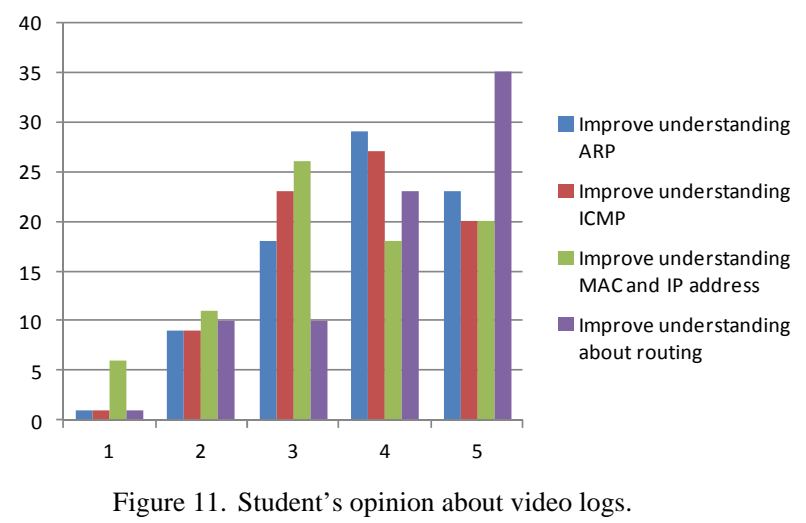

important questions have been consulted. The scale of qualification is between 5 (totally agree) and 1 (totally disagree) for the survey. The first four proposed questions are about what topics are easier to understand when the videos are seen by the students. The $65.1 \%$ (52 of 80 students) and $55.8 \%$ (47 of 80 students) think that they have now a better understanding of ARP and ICMP protocols, respectively. In these cases, the students have voted 4 or more when they have been asked. The same way, the $46.9 \%$ (38 of 81 students) and $73.4 \%$ (58 of 79 students) opined that the comprehension of addressing and routing is over TCP/IP are better after watching the videos.

With a fifth question the teachers want to know whether the videos are useful for the distance education as well for the classroom education. That is, the degree to which they could replace the teacher's explanations. In general, the $73 \%$ of students (58 of 80 respondents) give an affirmative answer when they are asked about this (they voted 4 or more).

\section{CONCLUSIONS}

This paper explains how the learning process is enhanced using a set of web 2.0 resources such as blogs, OCWs and video logs. Overall, this type of OERs is an effective tool for motivating the learning. In addition, the learning process becomes more informal. The blogs and OCW improve the search of resources while avoiding the dispersions of contents. Furthermore, the participation and collaborative relationships are increased, altruistically when blogs, OCWs, and video blogs are used. Students valuate positively the added value of being able to communicate online with other people and the suggestions of links to other websites for finding other courses, software, simulators or educational resources. Thus, these tools help students find relevant contents and understand concepts.

Moreover, the students were asked if the teaching/learning model supplemented with these tools is better than a teaching/learning without them. The opinion survey consisted of 3 additional questions. In these questions, the teachers wanted to know if the students considered these educational resources (blogs, OCW and video logs) essential tools to learn Computer Networks subject and therefore if they thought that it should be added to other subjects of the academic course and/or degree. A comparison of these three opinions demonstrates the acceptance level of the new resources to the learning process by the students (Fig. 11). Analyzing the results in

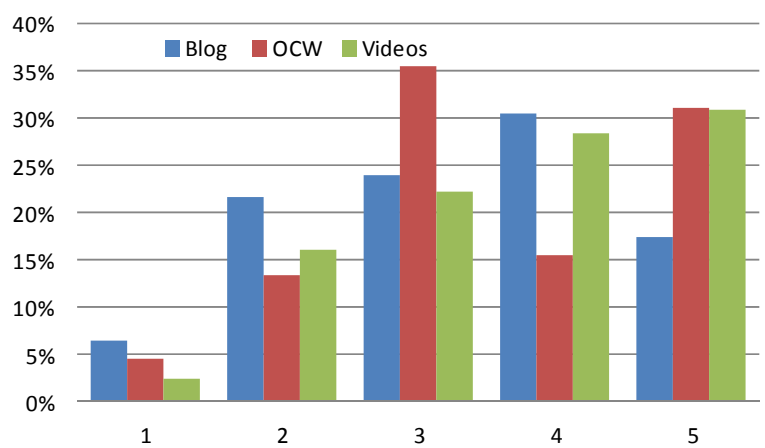

Figure 12. Are OCW, blog and videos any essential tools to improve the learning process in face to face methodology?

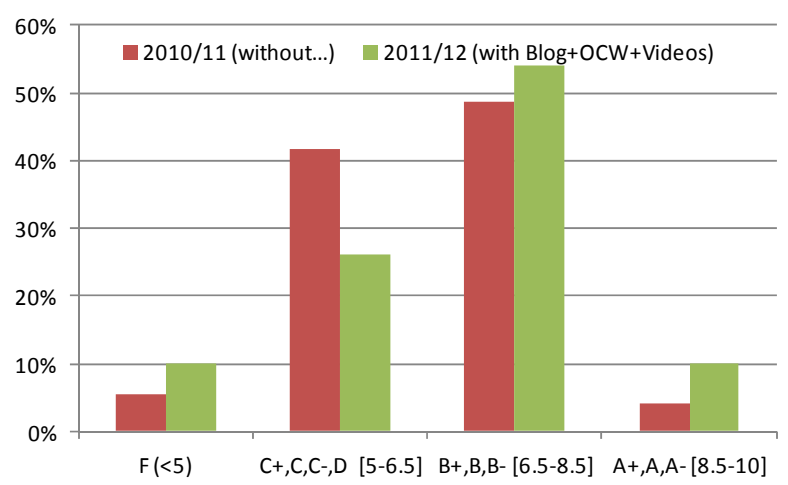

Figure 13. Grades acquired by students about 'Computer Network' subject.

detail, the $71.7 \%, 82.3 \%$ and $81.5 \%$ of 80 students have voted 3 or more in a scale between 1 and 5. Moreover, $47.8 \%$ of students have voted 4 or more versus $28.2 \%$ of students which have voted 2 or less when they are asked about Blogs. The results are even better when the opinion is about OCW or Blogs. In these cases are $46.7 \%$ versus $17.7 \%$ and $59.3 \%$ versus $18.5 \%$, respectively. For this reason, the majority opinion indicates that these resources begin to be essential in the future of education.

Analyzing the results of students during the past two academic courses (2010/11 and 2011/12), it can be deduced that the efficacy of learning is better when blogs, OCW and videos have been used as educational support (Fig. 12). Fig. 12 shows how the students have obtained higher grades when they have used these new educational resources. In general, there are more students with a success rate greater than 6.5 of 10 points. At the same time, the number of students who achieved an excellent level of knowledge has increased.

\section{ACKNOWLEDGMENT}

This work is supported by the "Education Science Institute" and the "Technology \& Educational Innovation Vice-President Office" of the University of Alicante through the aid "Technologic \& Educative Research Groups (GITE)”.

\section{REFERENCES}

[1] T. Clausen, "Undergraduate engineering education challenged by the Bologna declaration,”. IEEE Trans. on Education, vol. 48(2), pp. 213-215, May 2005. http://dx.doi.org/10.1109/TE.2005. $\underline{846045}$

[2] A. Koohang, K. Harman, "Advancing sustainability of open educational resources”. Informing Science and Information Technology, vol. 4. pp. 535-544, 2007. 
[3] P. Gil, F.A. Candelas and C.A. Jara, "Constructive learning for networks courses based on compact simulations and SCORM" 2nd IEEE Int. Conf. on Engineering Education, pp. 110-115, April 2011, Amman (Jordan).

[4] P. Gil, F.A. Candelas and C.A. Jara, "Computer networks elearning based on interactive simulations and SCORM”. Int. J. of Online Engineering, vol. 7(2), pp. 15-23, 2011.

[5] MIT OpenCourseWare's home page, 2011, http://ocw.mit.edu/index.html, retrieved July 30, 2011.

[6] K.B. Yue, T.A. Yang, W. Ding, P. Chen, "Open courseware and computer science education”. J. of Computing Sciences in Colleges, vol. 20(1), pp. 178-186, October 2004.

[7] OCW-UA 'Computer network' subject, http://ocw.ua.es/ingenieria-arquitectura/redes/Course_listing, retrieved July 30, 2011

[8] R. Ahmad, W. G. Lutters, "Promoting reflective learning: the role of blogs in the classroom", Online Communities and Social Computing. LNCS, vol. 6778, pp. 3-11, 2011. http://dx.doi.org/10.1007/978-3-642-21796-8_1

[9] H.N. Kim, "The phenomenon of blogs and theoretical model of blog use in educational contexts", Computers \& Education, vol. 51, pp. 1342-1352, 2008. http://dx.doi.org/10.1016/j.compedu. 2007.12.005

[10] J. A. Larusson, R. Alterman, "Wikis to support the collaborative part of collaborative learning”, Computer-Supported Collaborative Learning, vol. 4, pp. 371-402, 2009. http://dx.doi.org/10.1007/s11412-009-9076-6

[11] Blog 'Computer network' subject, http://blogs.ua.es/redesitis, retrieved July 30, 2011.

[12] P. Gil, F.A. Candelas, J. Pomares, S.T. Puente, J.A. Corrales, C.A. Jara and et al., "Using Moodle for an automatic individual evaluation of student's learning," 2nd Int. Conf. on Computer Supported Education, pp. 189-194, April 2010, Valencia (Spain).

[13] Institutional Repository of the University of Alicante. http://rua.ua.es/en/, retrieved November 2011.

[14] A. Luehmann and L. Tinelli, "Teacher professional identity development with social networking technologies: learning reform through blogging," Educational Media International, vol. 45, pp. 323-333, 2008. http://dx.doi.org/10.1080/09523980802573263

[15] D. Churchill, "Educational applications of Web 2.0: Using blogs to support teaching and learning," British J. of Educational Technology, vol. 40, pp. 179-183, 2009. http://dx.doi.org/10.1111/ j.1467-8535.2008.00865.x

[16] H.-M. Lai and C.-P. Chen, "Factors influencing secondary school teacher's adoption of teaching blogs," Computers \& Education, vol. 56, pp. 948-960, 2011. http://dx.doi.org/10.1016/j.compedu. 2010.11.010

[17] F. A. Candelas, P. Gil, "Practical experiments with KivaNS: A virtual laboratory for simulating IP routing in computer networks subjects”. Research Reflections and Innovations in Integrating ICT in Education, vol. 3, pp. 1415-1418, 2009.

[18] S.Hrastinski, "A theory of online learning as online participation”, Computers \& Education, vol. 52, pp. 78-82, 2009. http://dx.doi.org/10.1016/j.compedu.2008.06.009

\section{AUTHORS}

P. Gil is with the Department of Physics, System Engineering and Signal Theory, University of Alicante, Carretera de San Vicente del Raspeig s/n, 03690 San Vicente del Raspeig, Alicante, Spain (e-mail: pablo.gil@ua.es). He received the Ph.D. degree in 2008, and currently he is Associate Professor. He teaches courses in computer networks and robot vision. His research interests include virtual laboratories, educational technologies and computer vision for robotic systems. He is member IEEE- Education Society, and Spanish Committee of Automatics (CEA).

F. A. Candelas is with the Department of Physics, System Engineering and Signal Theory, University of Alicante (UA), Carretera de San Vicente del Raspeig s/n, 03690 San Vicente del Raspeig, Alicante, Spain (e-mail: francisco.candelas@ua.es). He received the Ph.D. degree in 2001, and currently he is Associate Professor. His main researching interest topics are virtual and remote laboratories, industrial automation and computer networks. He also belongs to Spanish Committee of Automatics (CEA) since 1999.

G. J. García is with the Department of Physics, System Engineering and Signal Theory, University of Alicante, Carretera de San Vicente del Raspeig s/n, 03690 San Vicente del Raspeig, Alicante, Spain (e-mail: gjgg@ua.es). He received the Ph.D. degree in 2010, and currently he is Assistant Professor. His research interests include educational technologies and robot vision. He belongs to Spanish Committee of Automatics (CEA) since 2004.

C. A. Jara is with the Department of Physics, System Engineering and Signal Theory, University of Alicante, Carretera de San Vicente del Raspeig s/n, 03690 San Vicente del Raspeig, Alicante, Spain (e-mail: carlos.jara@ua.es). He received the Ph.D. degree in 2010, and currently he is Assistant Professor. His research interests include virtual and remote laboratories, collaborative learning, robotics and industrial automation. He belongs to Spanish Committee of Automatics (CEA) since 2006.

This work was supported in part by the Education Science Institute and the Technology \& Educational Innovation Vice-President Office of the University of Alicante through the aid "Technologic \& Educative Research Groups”. Manuscript received 28 May 2012. Published as resubmitted by the authors 7 August 2012. 